\title{
Reseña de Canto Ortiz (2019) Psicología de los grupos: Fundamentos teóricos para la práctica e intervención grupal
}

\author{
Anastasio Ovejero \\ Universidad de Oviedo
}

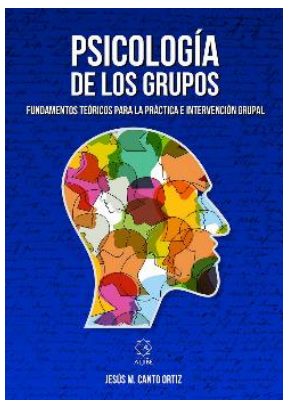

Canto Ortiz, Jesús M. (2019). Psicología de los grupos: Fundamentos teóricos para la práctica e intervención grupal. Málaga: Aljibe ISBN: 978-8497008662

El libro que pretendo reseñar es un libro bien escrito, claro, muy completo y muy actualizado, como corresponde a un libro de texto para el alumnado, pero a la vez nada simple, como corresponde a un texto universitario escrito por un catedrático de Psicología Social experto precisamente en el estudio de los grupos, tema en el que lleva trabajando muchos años. Es un libro bien planteado, bien diseñado y muy didáctico, lo que es enormemente importante en este contexto. Y su contenido ofrece una lista muy completa de los fenómenos grupales más importantes. En efecto, además de una interesante Introducción, consta estos nueve capítulos:

1.- Antecedentes históricos de la psicología de los grupos. El estudio de los grupos en psicología social

2.- Definición y tipos de grupos

3.- Formación y desarrollo del grupo
4.- La estructura del grupo
5.- El liderazgo en los grupos
6.- Influencia social en los grupos
7.- Toma de decisión en grupo
8.- Productividad y rendimiento grupal
9.- Relaciones intergrupales

Leyendo este libro, se percibe fácilmente la mucha experiencia de su autor como docente e investigador en este campo. A pesar de que, como ya he dicho, se trata de un manual, sin embargo, fácilmente se ve que es una obra de madurez de su autor. Ello se constata, por ejemplo, en la forma en que, para su mejor comprensión, relaciona e interacciona unos temas con otros. Así ocurre, por ejemplo, cuando al explicar que no puede existir un grupo social sin interacción, ni hay interacción sin comunicación, Jesús Canto pone de relieve la estrecha relación entre la interac- 
ción social y la comunicación dentro de los grupos y la crucial importancia que tiene la comunicación entre los miembros del grupo para la propia existencia de éste y, por supuesto, para su buen funcionamiento.

Un aspecto fundamental de esta obra es lo claro que deja que el estudio de los grupos es algo fundamental para entender la conducta humana y que es tan importante para la Psicología Social, que ésta nació, tal como señala Canto, como un intento de resolver "el problema teórico del individuo, del grupo y de la sociedad". Se trataba de resolver si el comportamiento del grupo implicaba procesos psicológicos y sociales irreductibles a las propiedades de los individuos, o si, por el contrario, los procesos eran exclusivamente individuales. La cuestión planteada tenía que ver con la propia definición de la psicología social y se convirtió en la controversia central de esta disciplina. En un principio, se llegó a identificar la Psicología Social con la Psicología de los Grupos, ya que los primeros psicólogos sociales trataban casi con exclusividad de la relación individuo-grupo. No era fácil, pues, la distinción entre ambas disciplinas, "ya que lo grupal era percibido y tratado como un requisito indispensable para una psicología auténticamente social" (2019, pp. 1718). No olvidemos, como subrayan Hogg y Moreland (1993), que casi cualquier fenómeno que estudia la Psicología Social (autopercepción, formación y cambio de actitud, atracción interpersonal, influencia social, conformidad y desviación, prejuicio, etc.) sucede en el contexto de los grupos pequeños. $Y$ tampoco podemos olvidar, como nos recuerda el propio Canto, que los grupos constituyen un fenómeno universal en el sentido de ser transhistóricos y transculturales (Stangor, 2004). Comprender y explicar la realidad de los grupos, añade, es esencial para entender y explicar la conducta humana. De hecho, no se puede entender con precisión la conducta de cualquier individuo sin atender a su contexto grupal (Mead, 1966/1934). De ahí la enorme importancia de conocer los grupos y lo que pasa dentro de ellos para entender a los seres humanos, su conducta y las relaciones interpersonales.

Como defiende Linnda Caporael (2007), y por decirlo con palabras de Jesús Canto, son muchos los factores que muestran que el ser humano es ante todo un ser grupal: 1) por sus características físicas, el ser humano ha necesitado siempre vivir en grupo para poder sobrevivir; 2) para que un grupo pueda existir y reportar beneficios a los individuos debe cumplir ciertos requisitos de organización interna que hagan la conducta de los miembros previsible y coordinada; 3) estos requisitos crean limitaciones sistemáticas para la adaptación biológica y psicológica de los individuos; esto significa que el grupo es el ambiente selectivo por antonomasia para el ser humano, donde ha evolucionado nuestra especie; y 4) como consecuencia de lo anterior, lo que interesa buscar son mecanismos individuales que hayan permitido desarrollar y mantener la pertenencia al grupo y mecanismos grupales que hayan permitido la conducta grupal frente al individualismo.

Por consiguiente, no es extraño que la Psicología Social naciera muy vinculada al interés por los grupos y a su preocupación por los retos que planteaba la nueva dinámica grupal de la modernidad, del proceso de progresiva urbanización y de la actividad sindical (movilizaciones, huelgas, etc.). Y lo hizo en primer lugar en Europa como psicología del comportamiento colectivo (Ovejero, 1997): los empresarios salidos de la revolución industrial necesitaban una nueva herramienta para combatir el empuje sindical, sobre todo en Francia (Tarde, 1986/1898; Le Bon, 1983/1895) y en Italia, donde podemos decir que realmente nació la Psicología Colectiva (Cattaneo, 1859; Orano, 1902; Sighele, 1892). En Alemania las cosas iban por otros derroteros, pero que no dejaban de estar muy relacionados con los grupos: la psicología de los pueblos (Lazarus y Steinthal, 1860; Wundt, 1920; véase Ovejero, 1994). Pero fue en Estados Unidos, donde estaban más preocupados por los pequeños grupos que por los movimientos de masas, donde hay que colocar el auténtico origen de la psicología social tal como la conocemos hoy día, que es la que se desarrolló a lo largo de todo el siglo XX: siempre con los grupos como elemento central de la disciplina, aunque casi exclusivamente los grupos pequeños.

Por consiguiente, no es de extrañar que el estudio de los grupos humanos no sólo estuvo en el origen de la psicología social, sino que el campo de la psicología de los grupos desempeñó siempre -y lo sigue desempeñando- un papel fundamental en el desarrollo de la Psi- 
cología Social. No podía ser de otra manera dado que cuando decimos que el ser humano es ante todo un ser social, queremos decir especialmente que es un ser grupal, que es a través de su interacción dentro de diferentes grupos (familia, grupo de amigos, compañeros de clase y de trabajo, etc.) como pasa de ser el organismo puramente biológico que era al nacer, a ser la persona que es ahora. Durante gran parte de nuestra vida, las personas no podemos desarrollarnos - ni siquiera sobrevivir- sin la ayuda de otras personas, sin el grupo o grupos a que pertenecemos; y durante toda nuestra vida nuestro desarrollo intelectual, nuestro bienestar psicológico, nuestra felicidad y, en general, toda nuestra conducta, depende principalmente de los grupos a los que pertenecemos y del papel que desempeñamos dentro de cada uno de ellos. Por eso precisamente, dice Canto al comienzo de la Introducción (p. 13):

El grupo no es una opción para el ser humano, es su condición. La mayor parte del tiempo las personas lo pasan en grupos; los acontecimientos más importantes de sus vidas suceden en grupos; su propia psicología es conformada a través de los grupos de los que forma parte. Además, la sociedad tiene a los grupos como principales agentes de socialización, como lugar idóneo para la influencia social que refleja un contexto histórico y cultural determinado. No se entiende al ser humano si no se presta atención a los grupos de los que ha formado parte. El proceso de convertirse en persona es una experiencia social en la que el grupo ocupa un lugar privilegiado; a través del grupo la sociedad se filtra y la mente se conforma.

Ahora bien, a través del análisis de la Psicología de los Grupos -y de los numerosos y diversos fenómenos que engloba (cohesión grupal, liderazgo, facilitación y holgazanería sociales, polarización grupal, pensamiento de grupo, etc.) - este libro viene a ser también un compendio de Psicología Social, de tal forma que algunos de los más importantes protagonistas de esta disciplina ocupan un lugar destacado en este libro: Lewin, Asch, Milgram, Sherif... Por otra parte, dado que Canto trata también una serie de temas que no son exclusivos de la Psicología de los Grupos, como es el caso de la obediencia a la autoridad o el de la influencia social, este texto viene a ser también un manual de Psicología Social hecho desde una perspectiva nada individualista, sino abiertamente psicosocial, en este caso grupal, que es una forma privilegiada de manifestarse lo psicosocial, lo que es a la vez psicológico y social. Al fin y al cabo, no sólo no existe ninguna frontera entre la Psicología Social y la Psicología de los Grupos, es que la Psicología Social no puede prescindir de la Psicología de los Grupos, ni siquiera alejarse de ella, pues, como señala el propio Canto (2019, p. 13), algo con lo que estoy completamente de acuerdo, el ser humano es un ser esencialmente grupal: la grupalidad no es algo añadido a la definición de lo que son los hombres y mujeres, sino algo constitutivo de su propia existencia. Tengamos muy presente que:

El individuo es bastante incomprensible si lo separamos de los grupos de los que forma parte, del mismo modo que no se puede comprender el grupo si queda separado de los individuos que lo componen y del contexto histórico-cultural en el que tiene lugar. De hecho, el grupo debe ser considerado como un mecanismo intermedio entre el individuo y la sociedad. (Canto, 2019, p. 14)

No olvidemos que, si es cierto y obvio que sin individuos no habría grupos ni sociedad, también es cierto -aunque menos obvio-que sin sociedad y sin grupos no habría individuos, no habría personas. Es la interacción social dentro de los grupos y las organizaciones lo que nos va convirtiendo en personas.

Por otra parte, este libro es de gran utilidad para quienes trabajan con grupos, sea en el ámbito laboral, como es el caso del trabajo en equipo, sea en el ámbito escolar, como es el caso especialmente del aprendizaje cooperativo en el aula, o sea en cualquier otro campo. La utilidad de la Psicología de Grupos para el citado caso escolar proviene, en primer lugar, del hecho, ya anunciado por Muzafer Sherif (1966), de que el concepto de interdependencia es crucial en la dinámica integrupal, tanto la negativa (en forma de conflictos de intereses) como la positiva (intereses positivos compartidos y metas supraordenadas), pero sobre todo la positiva, pues facilita la estructura grupal, la sensación de pertenencia y la reducción del conflicto intergrupal. Algo similar sostenía, a su manera, Kurt Lewin. No es por azar que ambos, Sherif y Lewin, estén en la base de los grupos de aprendizaje cooperativo (véase Ovejero, 1990, 2018), a causa precisamente de la importancia que conceden al concepto de interdependencia en los grupos. Al fin y al cabo, David Johnson, principal protagonista en la creación y difusión de los métodos de aprendizaje cooperativo, fue discípulo de Morton 
Deutsch, discípulo, a su vez, de Lewin. De hecho, el criterio de interdependencia, que tal vez sea el rasgo esencial de un grupo cooperativo de aprendizaje, ha sido el más aceptado a la hora de definir los grupos en Psicología Social.

Sin embargo, Solomon Asch desarrolló el concepto de grupo a partir de la interacción social y no a partir de la interdependencia. Pero ambas cosas, la interdependencia positiva y la interacción interpersonal, que son los dos principales requisitos para que haya grupo, son también los dos principales requisitos que debe tener un grupo para ser un grupo de aprendizaje cooperativo (Johnson y Johnson, 1990; Ovejero, 1990, 2018). Y no es raro que el aprendizaje cooperativo, entre sus muchos y muy positivos efectos, esté el de mejorar el bienestar psicológico de los miembros del grupo, entre otras razones porque satisfacen su necesidad de pertenencia. Como escribe Canto:

Los grupos que nos proporcionan sentido de pertenencia tienen efectos positivos sobre nuestra psicología. Los grupos imbuyen nuestra vida de significado. Nos hacen sentirnos distintos y especiales, eficaces y exitosos. Nos aumentan nuestra autoestima. Estos efectos pueden proteger nuestro bienestar cuando la autoestima es amenazada y estos efectos pueden ayudarnos a enfrentarnos a las consecuencias negativas de ser miembros de grupos devaluados. $(2019$, p. 78$)$

De hecho, Henri Tajfel (1984) sostiene que, como consecuencia de una comparación intergrupal sostenida, la superioridad relativa percibida del endogrupo sobre los demás grupos suele aumentar la autoestima, el bienestar y la salud mental de los miembros del grupo. Sin embargo, si una persona pertenece a un grupo poco valorado y percibido como inferior en alguna dimensión, entonces la comparación intergrupal negativa provocará con mucha probabilidad una amenaza a su bienestar psicológico. Por tanto, la pertenencia a un grupo no es condición suficiente para la mejora del bienestar psicológico de sus miembros: es necesario, además, que ese grupo sea percibido como algo positivo.

Muy relacionada con la necesidad de pertenencia, hasta el punto de que a veces se las identifica, está la necesidad de afiliación. La necesidad de pertenencia es el reflejo de la tendencia afiliativa. Según Craig Hill (1987), las personas se afilian principalmente por cuatro razones: 1) para obtener estimación positiva, teniendo muy en cuenta que, como muestran Marc Leary y Roy Baumeister (2000), el nivel de autoestima de una persona es una medida subjetiva de cuanto se le valora socialmente; 2 ) para recibir apoyo emocional; 3) para adquirir información sobre qué opinan y cómo se comportan otras personas y, así, evaluar sus propias opiniones y conductas comparándose con los demás; y 4) para conseguir la atención de otras personas y poder recibir atención y aprobación de ellas. Pero no todos los grupos ni todas las afiliaciones permiten a sus miembros alcanzar estos objetivos. Por tanto, como sostienen Katharine Greenaway, Tegan Cruwys, S. Alexander Haslam y Jolanda Jetten (2016), no son los grupos por sí mismos los que tienen un impacto sobre la salud, sino que es el sentido de identidad social que ofrecen a sus miembros lo que influye en su bienestar psicológico, pues es a través de la capacidad que tiene la identidad social de satisfacer las necesidades psicosociales (de pertenencia, de mantener la autoestima, de mantener el control y la necesidad de sentir que se tiene una existencia significativa) lo que provoca un efecto positivo sobre el bienestar psicológico y la salud (Greenaway et al., 2016; Jetten et al., 2017). El bienestar psicológico aumenta con el mantenimiento y el desarrollo de las identidades sociales, así como con la adquisición de nuevas identidades sociales (Jetten, Haslam y Haslam, 2012). Pero la identidad social puede ser también una fuente de estrés perjudicial para la salud y el bienestar psicológico, como cuando se pertenece a un grupo estigmatizado, cuando el grupo promueve conductas perjudiciales para la salud o cuando los individuos pertenecen a grupos que no les proporcionan apoyo social (Dingle, Stark, Cruwys y Best, 2015).

Otro aspecto interesante de los grupos, que Canto trata convincentemente y que también está muy relacionado con el aprendizaje cooperativo, es el de la eficacia del grupo para la reducción de los prejuicios. Para que se dé tal eficacia debe haber contacto directo entre los miembros de unos y otros grupos, pero cumpliendo ciertas condiciones básicas, entre las que destacan estas tres: los miembros de los distintos grupos tienen que tener el mismo estatus; debe haber un clima social favorable al contacto intergrupal; y la consecución de metas supraordenadas ha de conseguirse a través de algún tipo de cooperación 
intergrupal que origine algún grado de interdependencia entre los miembros y una disminución de la competición entre los mismos, condiciones que cumplen los grupos de aprendizaje cooperativo (Allport, 1954; Amir, 1976; véase Ovejero, 2018, Capítulo 8).

Particularmente interesante $-y$ también muy aplicable tanto al aprendizaje cooperativo como al trabajo en equipo en las empresases el Capítulo 5, titulado "El liderazgo en los grupos", estrechamente relacionado tanto con la influencia social como con el poder, donde el profesor Canto presenta una concepción muy interesante y muy fértil del poder, de un poder que, cuando es abusivo, deriva en un dominio o dominación contra aquellos sobre los que se ejerce. Más aún, señala Canto que el líder es la persona que más influye en el establecimiento y éxito de los objetivos del grupo, de forma que para entender el liderazgo se analiza el proceso de influencia interpersonal. Por eso sabemos que el líder consigue ejercer influencia sobre sus seguidores a causa del poder que tiene sobre ellos. Pero hay muchos tipos de poder, y por tanto hay también muchos tipos de influencia por parte del líder. De hecho, aunque con diferentes matices entre ellos, son muchos los autores que definen el liderazgo como la capacidad y acción de influir en los miembros del grupo. Como dice Canto (2019, p. 143), manteniendo una concepción del poder muy foucaultiana, siguiendo aquí a Tomás Ibáñez (2005):

Los psicólogos sociales han establecido una estrecha relación entre el concepto de poder y el de influencia social (Haslam, 2001). Se ejerce poder cuando se modifica la conducta de otra persona o personas, en contra de su voluntad o en contra de lo que sería su voluntad si fuera consciente de lo que ocurre. La expresión más sutil del poder es aquélla en la que el individuo dominado hace suyo los planes del dominante.

Y añade (pp. 142-143):

Los conceptos de poder, dominación y estatus están estrechamente relacionados entre sí, ya que se refieren a manifestaciones del control social. El poder es un fenómeno básicamente interactivo. De hecho, la acumulación de interacciones entre individuos con diferentes grados de poder da lugar a relaciones de dominio. $Y$ un conjunto de relaciones de dominio nos lleva a una estructura social con diferentes posiciones jerárquicas (estatus) y con un sistema normativo que satisface ciertas funciones. En un gran número de definiciones del poder, desde una perspectiva psicosocial, subyace la idea de que las diferencias de poder definen una relación social en la que una de las partes implicadas en la relación (grupo o individuo) tiene la posibilidad de imponer sus deseos sobre los de la otra parte gracias a los recursos con que cuenta, ya sean materiales o sociales (Fiske y Dépret, 1996; Haslam, 2001). La definición de poder, pues, implica que el que detenta el poder impone su voluntad contra los que aplica el poder. Esta concepción del poder, que posee un marcado carácter weberiano (Weber, 1974), se relaciona principalmente con las conductas de dominación y con las conductas de obediencia".

Porque no olvidemos que si unos mandan es porque otros obedecen.

También Ignacio Martín-Baró (1989) sostiene que el poder desempeña un papel muy importante tanto en la conformación del orden social como en la construcción de nosotros mismos, o sea, en la configuración de nuestra realidad personal e incluso en nuestra manera de pensar, de sentir y de comportarnos. El mayor poder que puede conseguir alguien es el que proviene de la internalización de la propia ideología por parte de aquellos sobre los que se ejerce influencia. De ahí deriva el gran poder actual de los neoliberales, que han conseguido ser hegemónicos a nivel planetario a causa de que la mayoría de la gente ha internalizado la ideología neoliberal (Ovejero, 2014).

Por tanto, tengamos muy presente que lo malo no es tanto el poder cuando el dominio, o sea, la utilización del poder para dominar a los demás miembros del grupo. Así, el acoso laboral constituye el ejercicio de la dominación sobre una persona por parte de alguien que tiene poder sobre ella. Pero ese poder se puede utilizar de otras maneras. El poder es la capacidad de ejercer influencia y, por tanto, puede ejercerse a en contra o a favor de alguien. Lo grave, por tanto, no es el poder, que es algo inevitable en los grupos humanos, cuanto la dominación, que sí es evitable y debe ser evitada.

También es un tema de gran utilidad para todos los que trabajan con grupos la llamada holgazanería social, que es la reducción de la aportación al rendimiento del grupo por parte de sus miembros, y que, como señala Canto (2019, p. 234):

Ocurre con más probabilidad cuando los miembros del grupo perciben que sus contribuciones no son identificables ni indispensables y cuando piensan que su contribución es desproporcionada en relación con la que realizan otros miembros del grupo [...] Cuando los miembros del grupo estiman que sus ejecuciones individuales son identificables y conocidas por los otros, la holgazanería 
social se reduce al máximo, porque los miembros del grupo abandonan la sensación de anonimato.

Pero hay mucho más, como demostraron las colectividades libertarias que se implantaron en la España de los años 30 allí donde fracasó el golpe de estado de los militares rebeldes (Ovejero, 2017): el grado de compromiso con la tarea y con el propio grupo reduce la probabilidad de que se dé holgazanería social en los grupos. Algo similar han demostrado también los métodos de aprendizaje cooperativo. La eficacia tanto de las colectividades libertarias como de los grupos de aprendizaje cooperativo proviene principalmente de sus rasgos grupales positivos: interacción positiva cara a cara, mucha atracción interpersonal, una buena interdependencia, cohesión grupal, metas ilusionantes y compartidas, etc., pero también a causa de otros factores como el hecho de que la adopción de roles directivos no son permanentes sino rotatorios; porque no hay miembros con un estatus superior y miembros con un status inferior, lo que tiene influencia incluso en la salud física de sus miembros, como claramente está mostrando la llamada Sociología Clínica, tan querida por José Ramón Torregrosa (2013) durante sus últimos años; y sobre todo por las relaciones interpersonales de amistad que existen dentro de los grupos cooperativos. Al fin y al cabo, como señalan Richard Wilkinson y Kate Pickett $(2009$, p. 65), son muchos los estudios que demuestran convincentemente los beneficios que para la salud tiene la amistad y la participación activa en la vida comunitaria.

En definitiva, estamos, pues, ante un libro muy interesante y fértil para entender a las personas y su comportamiento, y también para entender la propia Psicología Social y sus procesos. Pocos peros podría yo ponerle a este libro. Si acaso uno, y es que cuando su autor habla de la eficacia de los grupos no siquiera menciona, aunque es un tema que él conoce muy bien, las colectividades libertarias que se crearon en España entre 1936 y 1938: fueron cientos y cientos las comunidades que funcionaron en grupos cooperativos, con una autogestión prácticamente completa y con una eficacia realmente asombrosa, máxime si tenemos en cuenta que existieron en unas condiciones muy precarias y con la oposición frontal de todos los poderes económicos y estatales. Hubiera sido interesante que Canto hubiera insistido más en la importancia de la autogestión para mejorar la eficacia de los grupos, con las colectividades libertarias como ejemplo. $Y$ ello es algo extraño dado que Canto tiene una visión positiva de los grupos, frente a la tradicional visión negativa que a menudo ha reinado en psicología social y tiene también una opinión muy positiva de esas colectividades libertarias. Tengamos presente que la eficacia de tales colectividades se debió en gran medida a los procesos grupales que pusieron en marcha, dentro de un contexto autogestionario, como fue el trabajo en grupo, las decisiones tomadas en asambleas periódicas, el liderazgo compartido, una elevada cohesión de grupo, etc. (Ovejero, 2017). No olvidemos que, como hace ya muchos años señalara Muzafer Sherif (1936), "todo el mundo coincide en que la experiencia y la conducta del individuo es distinta cuando está solo que cuando se encuentra en grupo" (p. 56), sobre todo si el grupo tiene unas características muy concretas que hacen que surjan cualidades nuevas y estructuras psicológicas que son interiorizadas por sus miembros (Canto, 2019, p. 33), pues, como subraya el propio Sherif (1966, p. 75), "una situación psicológica grupal altera la percepción de los individuos y los productos grupales que resultan de ella son realidades importantes".

Estamos, pues, ante un libro muy útil para todas aquellas personas que se interesan por la Psicología Social e imprescindible para quienes están interesados en la Psicología de los Grupos, siendo especialmente fértil en campos en los que, como es el caso del aprendizaje cooperativo en el ámbito escolar o el trabajo en equipo en el ámbito laboral, los grupos son un elemento absolutamente esencial.

\section{Referencias}

Allport, Gordon (1954). The nature of prejudice. Cambridge, Mass.: Addison-Wesley.

Amir, Yehuda (1976). The role of intergroup contact in change of prejudice and ethnic relations. En Phyllis A. Katz (Ed.), Towards the elimination of racism (pp. 245-280). Nueva York: Pergamon Press.

Canto Ortiz, Jesús M. (2019). Psicología de los grupos: Fundamentos teóricos para la práctica e intervención grupal. Málaga: Aljibe.

Caporael, Linnda (2007) Evolutionary theory for social and cultural psychology. En E. Tony Higgins 
\& Ariel W. Kruglanski (Eds.), Evolutionary social psychology (pp. 3-18). Nueva York: Erlbaum.

Cattaneo, Carlo (1859). Psicologia delle mente associate. Turín: Giulio Einandi.

Dingle, Genevieve A.; Stark, C.; Cruwys, Tegan \& Best, D. (2015). Social identities as pathways into and out of addiction. Frontiers in Psychology, 6, 17951.

https://doi.org/10.3389/fpsyg.2015.01795

Fiske, Susan T. \& Dépret, Eric (1996). Control, interdependence and power: Understanding social cognition in its social context. European Review of Social Psychology, 7, 31-61.

https: / / doi.org/10.1080/14792779443000094

Greenaway, Katharine H.; Cruwys, Tegan; Haslam, S. Alexander \& Jetten, Jolanda (2016). Social identities promote well-being because they satisfy global psychological needs. European Journal of Social Psychology, 46, 294-307. https://doi.org/10.1002/ejsp.2169

Haslam, S. Alexander (2001). Psychology in organizations: The social identity approach. Londres: Sage.

Hill, Craig (1987). Afiliation motivation: people who need people... but in different ways. Journal of Personality and Social Psychology, 52, 10081018. https:// doi.org/10.1037//00223514.52.5.1008

Hogg, M.A. \& Moreland, R.L. (1993). Studing social process in small groups. British Journal of Social Psychology, 32, 107-110.

Ibáñez, Tomás (2005). Contra la dominación. Barcelona: Gedisa.

Jetten, Jolanda; Haslam, S. Alexander; Cruwys, Tegan; Greenaway, Katharine; Haslam, Catherine \& Steffens, Nikklas (2017). Advancing the social identity approach to health and wellbeing: Progressing the social cure research agenda. European Journal of Social Psychology, 47(7), 789-802.

https://doi.org/10.1002/ejsp.2333

Jetten, Jolanda; Haslam, Catherine C.; \& Haslam, S. Alexander (2012). The social cure: identity, health and well-being. Nueva York, Psychology Press.

Johnson David W. \& Johnson, Roger (1990). Cooperation and competition: Theory and research. Hillsdale, N.J.: Addison-Wesley.

Lazarus, M. \& Steinthal, H. (1860). Einleitende Gedanke über Völkerpsychologie. Zeitschrif für Völkerpsuchologie und Sprachwissenschaft, 1, 1 72.

Leary, Marc R. \& Baumeister, Roy F. (2000). The nature and function of self-esteem: Sociometer theory. En Mark P. Zanna (Ed.). Advances in Experimental Social Psychology (Vol. 32, pp. 1-62). San Diego: Academic Press.

Le Bon, Gustave (1983/1895). Psicología de las masas. Madrid: Morata.

Martín-Baró, Ignacio (1989). Sistema, grupo y poder: Psicología Social desde Centroamérica (II). San Salvador: UCA Editores.

Mead, George H. (1966/1934). Espíritu, persona y sociedad. Barcelona: Paidós.

Orano, P. (1902). Psicologia Sociale. Bari: Laterza i Figli.

Ovejero, Anastasio (1990). El aprendizaje cooperativo: Una alternativa eficaz a la enseñanza tradicional. Barcelona: PPU.

Ovejero, Anastasio (1994). Wilhelm Wundt: ¿fundador de la psicología experimental no social o de la psicología social no experimental? Revista de Historia de la Psicología, 15(1-2), 123-150.

Ovejero, Anastasio (1997). El individuo en la masa: Psicología del comportamiento colectivo. Oviedo: Ediciones Nobel.

Ovejero, Anastasio (2014). Los perdedores del nuevo capitalismo: Devastación del mundo del trabajo. Madrid: Biblioteca Nueva.

Ovejero, Anastasio (2017). Autogestión para tiempos de crisis: Utilidad de las colectividades libertarias, Madrid: Biblioteca Nueva.

Ovejero, Anastasio (2018). Aprendizaje cooperativo crítico: Mucho más que una eficaz técnica pedagógica. Madrid: Pirámide.

Sherif, Muzafer (1936). The psychology of social norms. Nueva York: Harper \& Brothers.

Sherif, Muzafer (1966). Group conflict and cooperation. Londres: Routledge and Kegan Paul.

Sighele, S Scipio (1892). La foule criminelle. París: Alcan.

Stangor, Charles (2004). Social groups in action and interaction. Nueva York: Psychology Press.

Tajfel, Henri (1984). Grupos humanos y categorías sociales. Barcelona: Herder.

Tarde, Gabriel (1986/1898). La opinión y la multitud. Madrid: Taurus.

Torregrosa, José R. (2013) “Algunas reflexiones (asistemáticas) a propósito de la Sociología Clínica" (Conferencia inédita) en ISA RC46 Clinical Sociology Conference, Madrid, marzo.

Weber, Max (1974). Economía y sociedad. México, DF: FCE. 
Wilkinson, Richard \& Pickett, Kate (2009). Desigualdad: Un análisis de la (in)felicidad colectiva. Madrid: Turner.
Wundt, Wilhelm (1920). Völkerpsychologie (10 vols.). Leipzig: Kröner.

\section{ANASTASIO OVEJERO}

Catedrático de Psicología Social y Doctor en Psicología. Departamento de Psicología. Universidad de Valladolid

\section{DIRECCIÓN DE CONTACTO}

tasiovejero@yahoo.es

\section{FORMATO DE CITACIÓN}

Anastasio Ovejero (2019). Reseña de Canto Ortiz (2019) Psicología de los grupos: Fundamentos teóricos para la práctica e intervención grupal. Quaderns de Psicologia, 21(1), e1518.

http://dx.doi.org/10.5565/rev/qpsicologia.1518 